\title{
Redes Neurais MLP e NARX Aplicadas na Previsão da Velocidade do Vento em Parques Eólicos do Estado da Bahia
}

\author{
Matheus de Paula, \\ Marilaine Colnago, \\ Wallace Casaca ${ }^{3}$ \\ Departamento de Engenharia de Energia, UNESP, Rosana
}

\begin{abstract}
Resumo. Nos últimos anos o uso de fontes renováveis de energia tem impactado diretamente no paradigma de geração energética devido à capacidade de produzir energia de forma limpa e sustentável. Neste contexto, a expansão da energia eólica, em especial, no nordeste brasileiro, tem correspondido com cerca de $85 \%$ da geração nacional nessa categoria. Assim, visando otimizar o planejamento energético e as políticas de segurança pública de geração de eletricidade, este trabalho endereça o problema da predição do vento em um dos parques eólicos do estado da Bahia. Mais especificamente, este estudo explora, a partir de ferramentas de Aprendizado de Máquina, uma base de dados disponível pela EPE (Empresa de Pesquisa Energética) do primeiro complexo eólico baiano. Desta forma, foram aplicados dois modelos de Redes Neurais: Multi Layer Percepton (MLP), e Nonlinear Auto-Regressive with Exogenous Input (NARX) para predizer a velocidade do vento. Estudos de validação foram conduzidos a fim de verificar o grau de assertividade dos modelos na tarefa predição, conforme discutido neste estudo.
\end{abstract}

Palavras-chave. Energia, energia eólica, Bahia, redes neurais, modelos não-lineares.

\section{Introdução}

Nos últimos anos tem se observado na indústria energética global uma forte ascensão das fontes renováveis. Dentre os principais sorvedouros de energia dessa categoria, o vento tem desempenhado papel de grande protagonismo, em especial devido à sua onipresença em praticamente quase toda a extensão do globo, além da sua produção ter um custo bastante acessível [1]. Com a crescente preocupação não só acerca da geração de energia sustentável, mas também da preservação ambiental, vários países têm realizado altos investimentos na construção complexos eólicos. No caso do Brasil, o Programa de Incentivo às Fontes Alternativas de Energia Elétrica (PROINFA), instituído pela Lei 10.438/2002, implantou, até 2011, um total de 119 empreendimentos dessa natureza, sendo que 41 destes

\footnotetext{
${ }^{1}$ matheus.paula@unesp.br

${ }^{2}$ marilaine.colnago@unesp.br

3 wallace@icmc.usp.br
} 
são formados por parques eólicos ${ }^{4}$. No epicentro dessa expansão, encontra-se o Nordeste brasileiro, sendo que $85 \%$ da produção nacional dessa forma de geração está presente na região, o que totaliza cerca de $12,2 \mathrm{GW}$ de capacidade instalada de energia eólica ${ }^{5}$. Essa geração é conduzida basicamente por meio de aerogeradores, cuja capacidade energética depende da densidade do ar, da área coberta das hélices, e da velocidade do vento, onde a energia mecânica proveniente do ar é transformada em energia elétrica para consumo.

Embora existam diversas vantagens quanto ao uso da energia eólica em comparação com demais fontes renováveis, há, por outro lado, a desvantagem dessa energia ser suscetível à altas variações na potência efetiva, causadas por características do local da coleta. Com isso, a previsão da velocidade do vento torna-se um ponto primordial nos planos nacionais de contingência energética. Outro grande benefício em predizer a dinâmica do vento é que tal fonte primária de energia é uma das mais abundantes na natureza, o que permite sua integração com outras fontes intermitentes de energia de modo a maximizar a eficiência energética de sistemas híbridos de geração [6].

Com base na problemática e motivações apresentadas acima, este trabalho objetiva explorar o problema de predição de vento em um complexo eólico de energia. A fim de delinear o problema e a análise dos resultados, a pesquisa concentra-se em investigar um dos mais importantes parques eólicos da Bahia. O estudo foi desenvolvido a partir de dois modelos de Aprendizado de Máquina (Redes Neurais), a saber: Multi Layer Percepton (MLP) e a Nonlinear Auto-Regressive with Exogenous Input (NARX).

\section{Metodologia}

O processo metodológico desta pesquisa leva em consideração os seguintes ítens:

- Plataforma: MATLAB (R2017a), a qual possui diversas ferramentas de suporte e toolboxes tanto para o aprendizado de máquina como para a análise dos dados.

- Base de Dados: A base de dados utilizada foi disponibilizada pela Empresa de Pesquisa Energética (EPE). A EPE é uma empresa pública vinculada ao Ministério de Minas e Energia, que presta serviços relacionados ao setor energético. A base possui 1224 registros (tabela, em formato xlsx) sobre as medições anemométricas do vento, além da temperatura, umidade do ar, e direção predominante dos ventos na forma de séries históricas (coletas diárias, excluindo-se domingos e feriados), onde cada registro corresponde à média diária de cada uma das variáveis supracitadas, no período de 01/Jan./2012 a 31/Mar./2016. O parque investigado foi o complexo eólico de Brotas de Macaúbas, BA.

- Análise Exploratória dos Dados: Para a análise do conjunto das features (características/grandezas) disponíveis, foi necessário realizar um estudo prévio da correlação entre as variáveis envolvidas. Desta forma, os modelos tornaram-se mais assertivos, sendo possível inferir a respeito das variáveis que mais influênciam na predição do vento.

\footnotetext{
${ }^{4}$ Dados da Agência Nacional de Energia Elétrica (PROINFA - Energia Eólica): http://aneel.gov.br

${ }^{5}$ Nordeste gera $85 \%$ da energia eólica do Brasil, $2018 . \quad$ Disponível em: https://www.letrasambientais.com.br/posts/nordeste-gera-85-da-energia-eolica-do-brasil.
} 
- Modelos Preditivos: Para realizar a predição do vento, foram utilizadas duas configurações de rede: a Multi Layer Percepton (MLP), e a Nonlinear Auto-Regressive with Exogenous Input (NARX). A MLP é uma rede de multicamadas clássica ajustada a partir de uma quantidade específica de neurônios. Já a rede NARX considera múltiplas camadas de uma rede MLP a partir de uma processo de realimentação. A rede prevê valores futuros de uma série temporal $y(t)$ a partir de seus valores anteriores, $y(t-k)$, além de históricos anteriores de uma série temporal auxiliar, $z(t)$ [4]. Com isso, é possível realizar um re-treinamento sistemático de forma a melhorar a capacidade preditiva da rede. Em ambos os modelos, foram empregados: os neurônios da camada de entrada e da intermediária, os neurônios de saída, função de ativação, critério de parada, a taxa de aprendizado e delay (realimentação da NARX) [4]. Assim, a partir da análise do Erro Quadrático Médio (MSE) e do Erro Percentual Médio Absoluto (MAPE), foi possível verificar a eficácia dos modelos.

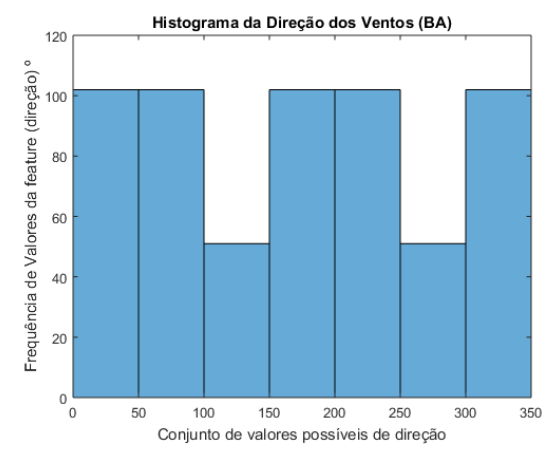

(a)

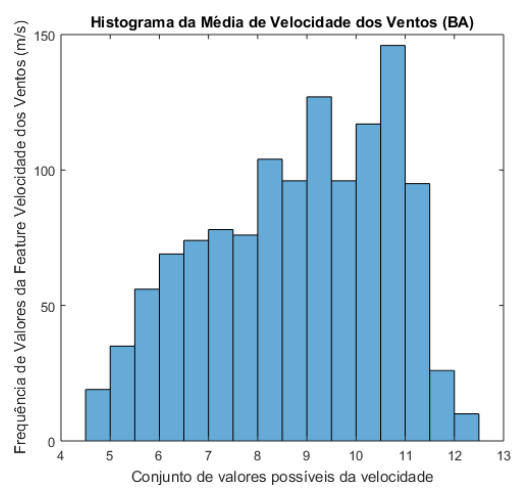

(c)

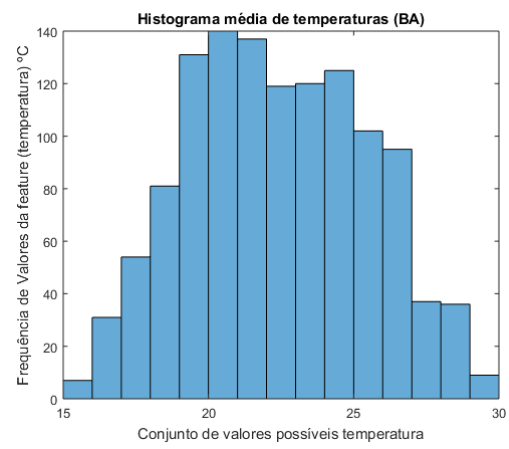

(b)

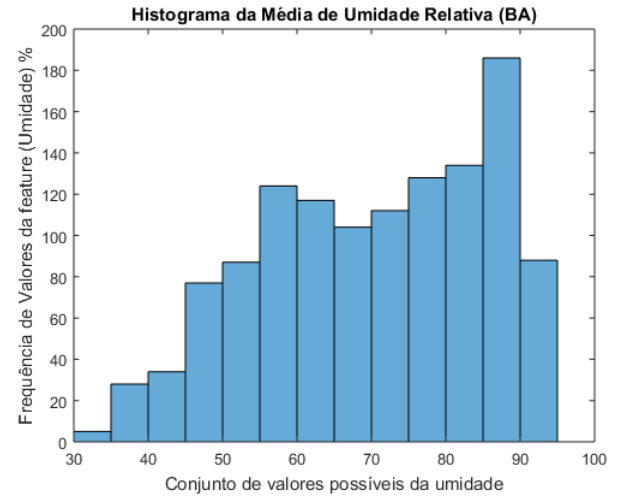

(d)

Figura 1: Histograma das features. (a) Direção. (b) Temperatura. (c) Velocidade. (d) Umidade.

\section{Resultados e Discussões}

Primeiramente, foi realizada a análise do comportamento das features disponíveis. Foram gerados histogramas para verificar a distribuição de cada variável (Figura 1). Além 


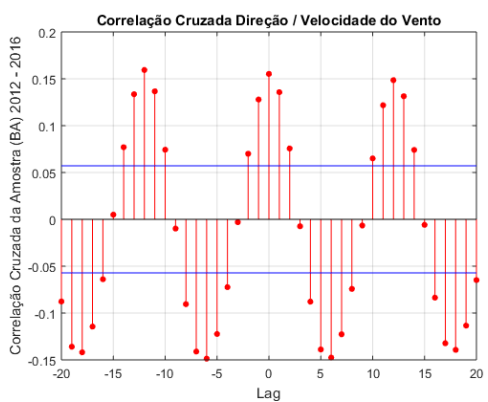

(a)

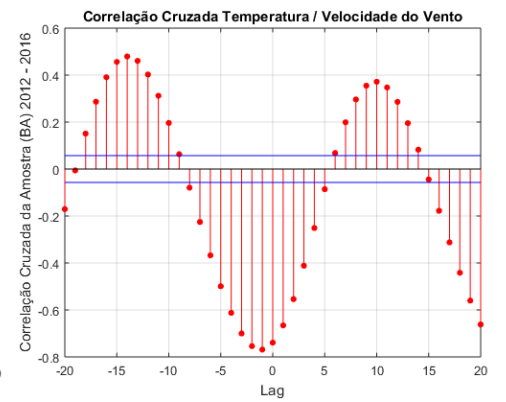

(b)

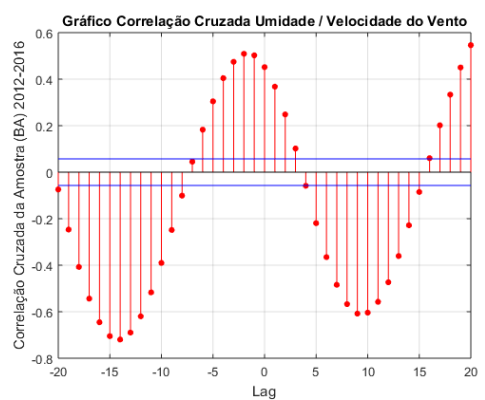

(c)

Figura 2: Correlação entre: (a) Direção, (b) Temperatura, (c) Umidade com relação à Velocidade.

disso, foi computada a correlação cruzada entre a velocidade do vento (variável target) versus temperatura, umidade do ar, e direção do vento (Figura 2), similar ao realizado em [3]. Com base nos gráficos originados, é possível identificar que o maior valor de correlação entre direção e velocidade fica na faixa de $0.15(\approx 0)$, o que nos leva a descartar a direção nos modelos de predição. Já na correlação da velocidade com a temperatura e a umidade, apesar da correlação em alguns lags (períodos de atraso de tempo), há também um grande número de correlações mais próximas de zero do que de um, o que nos motivou a desconsiderar o uso explícito dessas features nos preditores. Assim, foi adotada somente a velocidade do vento na modelagem preditiva do problema. Essa escolha também pode impactar positivamente na otimização da rede neural, conforme afirmam os autores de [5], que uma grande quantidade de entradas pode levar ao aumento desordenado no tamanho e na complexidade da rede, além da inclusão de entradas irrelevantes que poderiam afetar diretamente o processo de treinamento dos modelos.

$\mathrm{Na}$ sequência, foi considerado a velocidade do vento versus as coletas das médias horárias de vento (Figura 3), com o intuito de detectar variações bruscas no período de análise, além da correlação da própria velocidade do vento, em períodos consecutivos de 3 em 3 meses, totalizando um semestre completo (Tabela 1). Analisando a Figura 3, foi possível detectar que a amplitude varia no decorrer dos anos passados: em alguns períodos da amostra ocorrem pequenas taxas de ventos. Isso pode ser justificado pelo período de

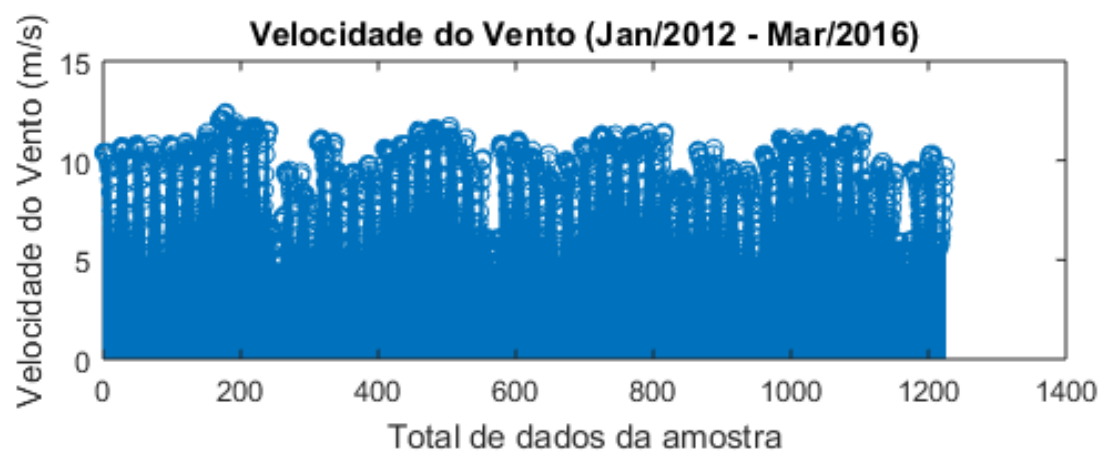

Figura 3: Plot da feature velocidade do vento versus coleta diária. 
declínio pós "safra dos ventos", que é o período mais propício para a ocorrência de ventos no nordeste. Já com relação aos resultados da Tabela 1, nota-se que nos anos de 2012 e 2013 (segundo semestre), ocorreram variações bruscas no fluxo do vento devido, provavelmente, ao término da safra dos ventos, embora esse não seja o comportamento predominante para o semestre, já que, em 2014 e 2015, atingiu-se uma alta correção.

Tabela 1: Correlação da feature velocidade do vento na amostra.

\begin{tabular}{ccccc}
\hline Meses/Anos & 2012 & 2013 & 2014 & 2015 \\
\hline $1^{o}-6^{o}$ mês & 0.9159 & 0.75388 & 0.6654 & 0.5235 \\
$7^{o}-12^{o}$ mês & 0.2092 & 0.3891 & 0.7903 & 0.7485 \\
\hline
\end{tabular}

A partir da realização de toda a etapa de sumarização e do estudo prévio dos dados, foram realizados diversos testes com distintas configurações das redes neurais adotadas, MLP e NARX. Para o modelo MLP, foram consideradas 3 camadas de entrada ( 8 séries de entrada de velocidade do vento), além de 1 saída (velocidade do vento +1 ). Com isso, foram testadas 3 configurações de redes distintas, alterando-se os neurônios da camada intermediária de modo a obter o melhor resultado em relação à métrica de erro MSE (Tabela 2). Os parâmetros utilizados pelos modelos foram: três séries de velocidade do vento (neurônios de entrada), uma série como neurônio de saída, o algoritmo clássico de otimização de Levenberg-Marquarat [7] e, como função de ativação, o Tansig (entrada e camada oculta), e Purelin (saída) [2]. Como critério de parada, adotou-se a validação cruzada com taxa de aprendizado de 0.00001. Finalmente, para testar os modelos na prática, a base foi dividida em 2/3 para dados de treinamento (de Jan./2012 até Out./2014), e 1/3 para validação (Nov./2014 - Mar./2016).

Tabela 2: Erro quadrático médio (Modelo MLP).

\begin{tabular}{cc}
\hline Neurônios da Camada Intermediária & Erro quadrático médio (MSE) \\
\hline 5 & $5.18 \times 10^{-2}$ \\
10 & $4.12 \times 10^{-2}$ \\
15 & $3.8 \times 10^{-2}$ \\
\hline
\end{tabular}

Foi possível constatar, a partir dos scores tabulados, que quanto maior a quantidade de neurônios da camada intermediária utilizada, melhor foi o resultado apresentado pela rede. Porém, é válido ressaltar que há um ponto ótimo de ajuste de forma que, a partir desse limiar, pode ocorrer o fenômeno de "sobreajuste dos dados", onde a rede perde a capacidade de previsibilidade quando aplicada na prática. Na Figura 4(a) é apresentado o erro médio absoluto (MAPE) da melhor configuração obtida para o MLP (isto é, 15 neurônios na camada intermediária) para todos os registros disponíveis dos últimos três meses da base, onde é possível observar que o referido modelo atinge um bom nível de assertividade. Isso se deve, principalmente, ao fato da velocidade do vento ter apresentado uma boa correlação em diversos períodos específicos de tempo ao longo dos anos.

Também foram realizadas três avaliações com a rede NARX (Tabela 3), relacionando 
os dados de entrada-saída e a saída prevista [4] com os mesmos parâmetros da MLP, porém, com um delay (realimentação) de 5 atrasos temporais. Para comparar diferentes configurações da rede, alterou-se novamente os neurônios da camada intermediária - tal como no caso anterior - com o intuito de atingir o menor erro quadrático médio. Os demais parâmetros são os mesmos utilizados no modelo MLP.

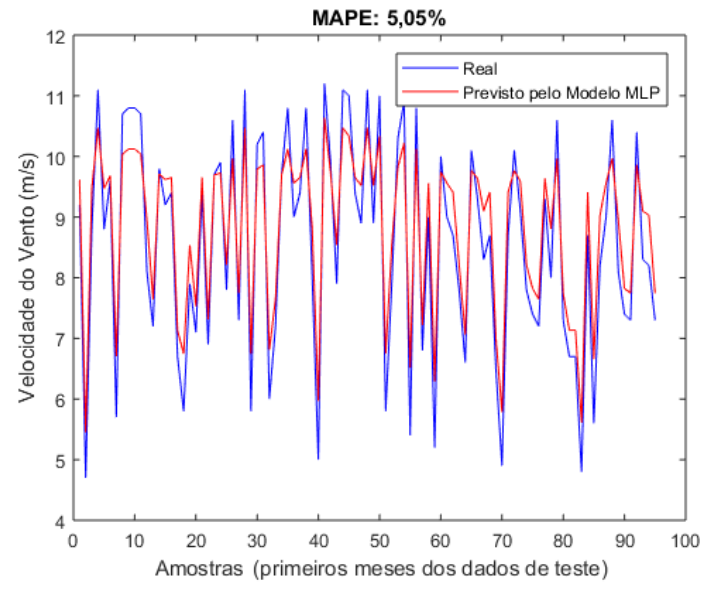

(a)

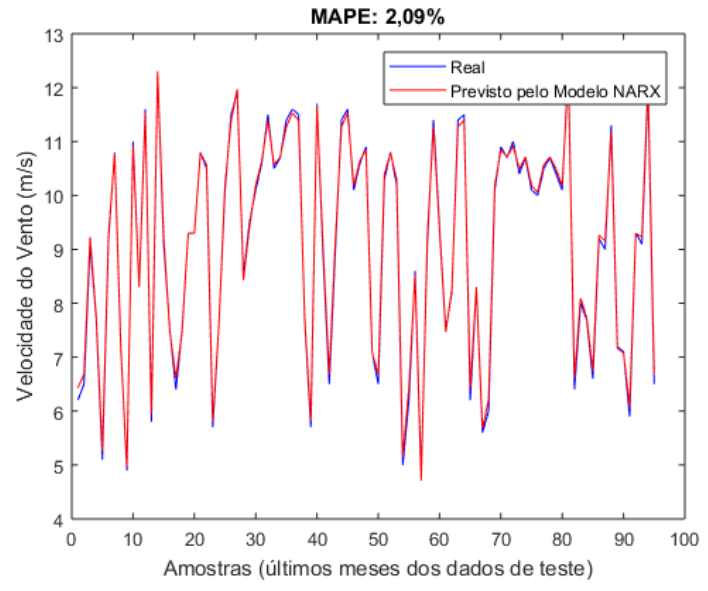

(b)

Figura 4: (a) Previsão do modelo MLP. (b) Previsão do modelo NARX. Curvas em azul fornecem os valores reais (para teste) enquanto que as curvas em vermelho representam os valores preditos.

Tabela 3: Erro quadrático médio (NARX).

\begin{tabular}{cc}
\hline Neurônios da Camada Intermediária & Erro quadrático médio (MSE) \\
\hline 5 & $2.3 \times 10^{-2}$ \\
10 & $2.2 \times 10^{-2}$ \\
15 & $2.1 \times 10^{-2}$ \\
\hline
\end{tabular}

A partir dos testes conduzidos acima, foi possível concluir que os neurônios da camada oculta não mudaram de forma radical o comportamento da aprendizagem na rede. Também, com base na Figura 4(b), foi possível constatar que a rede NARX alcança um excelente resultado em relação à mensuração do erro pelo MAPE. Finalmente, ao realizar uma análise comparativa entre os dois modelos de predição, é possível concluir que a rede NARX acaba por atingir uma maior capacidade de aprendizagem quando comparada à MLP (Tabela 4).

Tabela 4: Comparação entre as redes.

\begin{tabular}{ccc}
\hline Tipo de Rede & Erro quadrático médio (MSE) & Erro médio absoluto (MAPE) \\
\hline MLP & $3.8 \times 10^{-2}$ & $5.05 \%$ \\
NARX & $2.1 \times 10^{-2}$ & $2.09 \%$ \\
\hline
\end{tabular}




\section{Conclusão}

A partir do estudo conduzido, foi possível concluir que ambos os modelos são elegíveis para prever, com sucesso, a velocidade do vento no território baiano. Em termos mais pontuais, a análise exploratória dos dados foi de suma importância na tomada de decisões para otimizar as configurações das redes, além de permitir identificar padrões e analisar estatísticamente o comportamento das variáveis do problema. Na rede Multi Layer Percepton (MLP), foi possível observar que, após alguns testes aumentando-se os neurônios da camada oculta, a rede passou a não aprender mais, o que provavelmente pode ser atribuído ao fenômeno de "sobreajuste". Já em relação à rede NARX, o modelo apresentou resultados bastante acurados, mesmo com a variação da camada oculta, o que demonstra que tal modelo é bastante adequado para tratar o problema investigado.

Quanto à análise comparativa da assertividade dos modelos, foi possível constatar que a rede NARX apresentou resultados mais precisos com relação à MLP. Por fim, vale ressaltar que estudos nessa direção - da previsibilidade do vento para geração de energia eólica - são cada vez mais necessários para a elaboração e execução dos planos nacionais de geração e distribuição de energia, uma vez que permitem otimizar e melhor gerir o uso da energia, além de minimizar custos operacionais.

\section{Agradecimentos}

Os autores agradecem à FAPESP (Proc. \#2018/05341-4) pelo fomento à pesquisa.

\section{Referências}

[1] R. Aldabó, Energia Eólica. ArtLiber, 2002.

[2] X. Glorot and Y. Bengio, Understanding the Difficulty of Training Deep Feedforward Neural Networks. Proceedings of the 13th International Conference on Artificial Intelligence and Statistics, p. 249-256, 2010.

[3] G. C. Rodrigues, Utilização de Redes Neurais para Previsão de Ventos no Horizonte de 24 Horas. Dissertação de Mestrado, UFRJ, Rio de Janeiro, 2007.

[4] T. F. M. dos Santos, Aplicação de Séries Temporais e Redes Neurais em um Ambiente de Computação em Nuvem. Dissertação de Mestrado, UFSM, Santa Maria, 2014.

[5] M. L. P. Freire, Utilização das Redes Neurais na Previsão da Velocidade do Vento. Trabalho de Conclusão de Curso, Universidade de Pernambuco, Recife, 2014.

[6] T. T. D. Tran and A. D. Smith, Stochastic Optimization for Integration of Renewable Energy Technologies in District Energy Systems for Cost-Effective Use. Energies, v. 2, n. 3, p. 1-26, 2019.

[7] H. Yu and B. M. Wilamowski, Levenberg-Marquardt Training. Industrial Electronics Handbook, v. 5, p. 1-16, 2011. 